\title{
Aromatic Compounds from the Marine-Derived Fungus
} Aspergillus versicolor

\author{
Rong Yao $\oplus^{1,2}{ }^{*}$, Shen Yao $\oplus^{1}$ and Ailian Hua $\odot{ }^{1}$ \\ ${ }^{I}$ Department of Pharmacy, The First People's Hospital of Yuhang District, Hangzhou, Zhejiang \\ 311100, China \\ ${ }^{2}$ School of Pharmacy, Zhejiang University of Technology, Hangzhou 310014, China \\ (Received May 21, 2020; Revised June 06, 2020; Accepted June 12, 2020)
}

\begin{abstract}
Chemical investigation of the EtOAc extract of a marine-derived fungus Aspergillus versicolor SZW5 led to the isolation of one new compound, named aspeverether A (1), and seven known compounds. The structures were determined by extensive analyses of spectroscopic data (1D and 2D NMR, and HRESIMS). Compounds 1 and 3 possessed an unusual 1,3-dimethoxy-2-vinylbenzene moiety, which is rarely found in nature. Compound 6 showed strong inhibitory effect toward $\alpha$-glucosidase with an $\mathrm{IC}_{50}$ value of $71 \mu \mathrm{M}$, being more active than the positive acarbose $(210 \mu \mathrm{M})$.
\end{abstract}

Keywords: Aspergillus versicolor; aspeverether A; structure elucidation; marine. (C) 2020 ACG Publica-tions. All rights reserved.

\section{Plant Source}

Fungus SZW-5 was isolated from the sediments that were collected at a depth of $-3.5 \mathrm{~m}$ from the Shenzhen River Estuary. The strain was identified as Aspergillus versicolor by analysis of the internal transcribed spacer (ITS) region of the rDNA sequence. The ITS sequence has been submitted to the GenBank data base (http://www.ncbi.nlm.nih.gov) with the accession number MG845247. The strain SZW-5 was deposited at the Marine Culture Collection of China.

\section{Previous Studies}

In recent years, marine-derived fungi have attracted much attention from natural medicinal chemists, and many secondary metabolites possessing intriguing structures and various activities have been reported. The fungus A. versicolor has been proved to be prolific. Previous chemical study of marine-derived A. versicolor led to the identification of many secondary metabolites, including alkaloids [1-3], cyclopeptides [4-6], polyketides [7, 8], and depsides [9, 10], some exhibited pronounced biological activities, such as cytotoxic [2], antimicrobial [1], lipid-lowering [6], and DPPH radical scavenging effects [10]. In our study of bioactive secondary metabolites of a marine-

\footnotetext{
*Corresponding author: E- Mail: yaorong1205@163.com (R. Yao).
} 
derived fungus A. versicolor SZW-5, eight aromatic compounds including a new one were obtained (Figure 1). Herein, the isolation, structural identification, and the inhibitory effects toward $\alpha$ glucosidase of the metabolites were described.

\section{Present Study}

The solid-state fermentation was carried out in 15 erlenmeyer flasks $(500 \mathrm{~mL})$ with $70 \mathrm{~g}$ of rice and artificial sea-water $(90 \mathrm{~mL})$, and the contents were autoclaved at 15 psi for $30 \mathrm{~min}$. Each flask was inoculated with $3.0 \mathrm{~mL}$ of the spore inoculum and incubated at room temperature for 35 days. The fermented materials were extracted with EtOAc $(3 \times 2000 \mathrm{~mL})$ and to give an EtOAc extract $(3.1 \mathrm{~g})$, which was subjected to ODS silica gel column chromatography (CC) eluted with $\mathrm{MeOH} / \mathrm{H}_{2} \mathrm{O}(20: 80$ to100:0) to afford five fractions (F1-F5). F3 was chromatographed over ODS silica gel CC $\left(\mathrm{MeOH} / \mathrm{H}_{2} \mathrm{O}: 20: 80\right.$ to100:0) to give six fractions (F3a-F3f). F3a was purified by HPLC using $\mathrm{CH}_{3} \mathrm{CN} / \mathrm{H}_{2} \mathrm{O}(28: 72,3 \mathrm{~mL} / \mathrm{min})$ as eluent to afford $4\left(3.2 \mathrm{mg}, \mathrm{t}_{\mathrm{R}} 28.1 \mathrm{~min}\right)$ and $\mathbf{5}\left(1.9 \mathrm{mg}, \mathrm{t}_{\mathrm{R}} 33.2 \mathrm{~min}\right)$. F3c was separated by ODS silica gel $\mathrm{CC}$ eluting with $\mathrm{MeOH} / \mathrm{H}_{2} \mathrm{O}$ (20:80 to 100:0) to give six fractions (F3d1-F3d6). F3d2 was separated by $\mathrm{HPLC}\left(\mathrm{MeOH} / \mathrm{H}_{2} \mathrm{O}=50: 50,3 \mathrm{~mL} / \mathrm{min}\right)$ to yield five fractions (F3d2a-F3d2e). F3d3c was purified by $\mathrm{HPLC}\left(\mathrm{CH}_{3} \mathrm{CN} / \mathrm{H}_{2} \mathrm{O}=41: 59,3 \mathrm{~mL} / \mathrm{min}\right)$ to afford 6 $\left(6.5 \mathrm{mg}, \mathrm{t}_{\mathrm{R}} 19.5 \mathrm{~min}\right)$. F5 was further chromatographed over ODS silica gel CC eluted with $\mathrm{MeOH} / \mathrm{H}_{2} \mathrm{O}(20: 80 \rightarrow 100: 0)$ to afford four subfractions (F5a-F5d). Purification of F5a by HPLC $\left(\mathrm{CH}_{3} \mathrm{CN} / \mathrm{H}_{2} \mathrm{O}=54: 46,3 \mathrm{~mL} / \mathrm{min}\right)$ gave $7\left(5.3 \mathrm{mg}, \mathrm{t}_{\mathrm{R}} 9.7 \mathrm{~min}\right)$ and $8\left(3.7 \mathrm{mg}, \mathrm{t}_{\mathrm{R}} 11.9 \mathrm{~min}\right) . \mathrm{F} 5 \mathrm{~d}$ was purified by $\mathrm{HPLC}\left(\mathrm{CH}_{3} \mathrm{CN} / \mathrm{H}_{2} \mathrm{O}=68: 32,3 \mathrm{~mL} / \mathrm{min}\right)$ to give $2\left(14 \mathrm{mg}, \mathrm{t}_{\mathrm{R}} 24.5 \mathrm{~min}\right), 3\left(8.8 \mathrm{mg}, \mathrm{t}_{\mathrm{R}} 22.2\right.$ $\mathrm{min})$, and 1 ( $\left.24 \mathrm{mg}, \mathrm{t}_{\mathrm{R}} 24.2 \mathrm{~min}\right)$.

Aspeverether A (1): light yellow oil; UV (MeOH) $\lambda_{\max }(\log \varepsilon) 299$ (3.81), 258 (4.22), 220 (4.39); ${ }^{1} \mathrm{H}$ and ${ }^{13} \mathrm{C}$ NMR data, see Table 1 ; positive ESIMS $\mathrm{m} / z 363.1561[\mathrm{M}+\mathrm{Na}]^{+}$(calculated for $\left.\mathrm{C}_{21} \mathrm{H}_{24} \mathrm{O}_{4} \mathrm{Na}, 363.1567\right)$.

$\alpha$-Glucosidase Assay: The $\alpha$-glucosidase inhibitory effect was assessed as described in the literature [11].

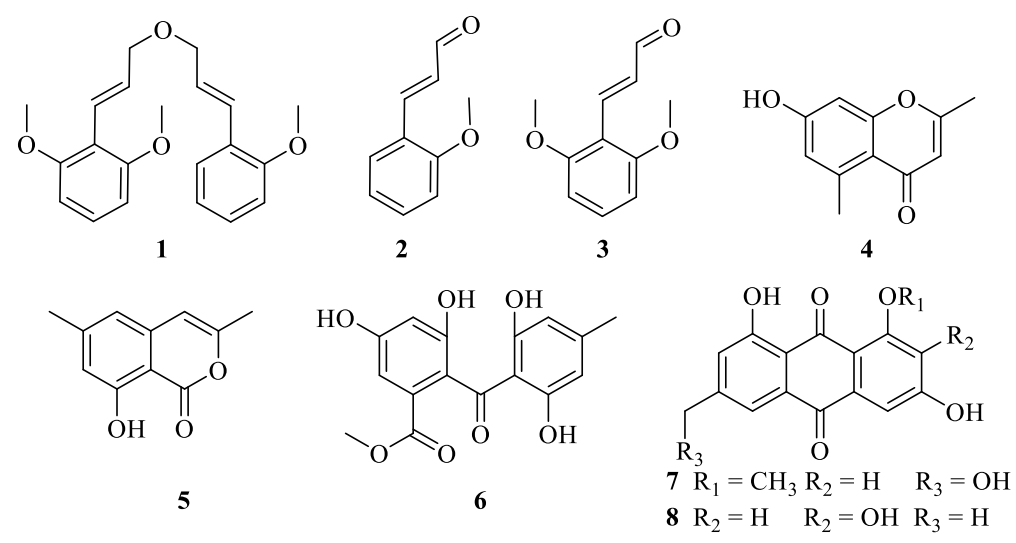

Figure 1. Structures of compounds 1-8 from A. versicolor SZW-5.

Compound 1, a light yellow oil, had the molecular formula $\mathrm{C}_{21} \mathrm{H}_{24} \mathrm{O}_{4}$ as established by the

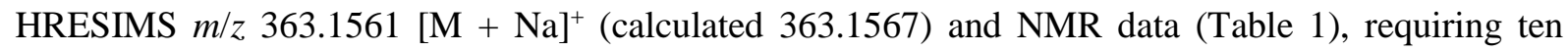
double bond equivalents. The ${ }^{1} \mathrm{H}$ NMR data exhibited the presences of three aromatic methoxys $\left(\delta_{\mathrm{H}}\right.$ $3.84,9 \mathrm{H})$, four olefinic protons $\left[\delta_{\mathrm{H}} 6.96(1 \mathrm{H}, \mathrm{d}, J=16.2 \mathrm{~Hz}), 6.76(1 \mathrm{H}, \mathrm{dt}, J=16.2,6.0 \mathrm{~Hz}), 6.38\right.$ $(1 \mathrm{H}, \mathrm{dt}, J=16.1,6.0 \mathrm{~Hz}), 6.98(\mathrm{~d}, J=16.1 \mathrm{~Hz})]$, a symmetrically $1,2,3$-trisubstituted benzene $\left[\delta_{\mathrm{H}} 6.65\right.$ $(2 \mathrm{H}, \mathrm{d}, J=8.4 \mathrm{~Hz}), 7.18(1 \mathrm{H}, \mathrm{d}, J=8.4 \mathrm{~Hz})]$, and a 1,2-disubstituted benzene $[6.98(1 \mathrm{H}, \mathrm{d}, J=7.8$ $\mathrm{Hz}), 6.99(1 \mathrm{H}, \mathrm{t}, J=7.8,7.6 \mathrm{~Hz}) ; 7.24(1 \mathrm{H}, \mathrm{td}, J=7.8,1.5 \mathrm{~Hz}) ; 7.51(1 \mathrm{H}, \mathrm{dd}, J=7.6,1.5 \mathrm{~Hz})]$ (Table 1). The ${ }^{13} \mathrm{C}$ NMR spectrum indicated the presences of twenty-one carbon resonances, including sixteen 
aromatic carbons for two benzenes and two double bonds, together with three methoxys $(\delta 56.1,56.1$, $55.8)$, and two oxymethylenes $\left(\delta_{\mathrm{C}} 73.0,71.4\right)$ (Table 1$)$.

Table 1. ${ }^{1} \mathrm{H}(400 \mathrm{~Hz})$ and ${ }^{13} \mathrm{C}$ NMR $(100 \mathrm{~Hz})$ Data of $\mathbf{1}$ in Acetone-d $\mathrm{d}_{6}(\delta$ in ppm $)$

\begin{tabular}{cccccc}
\hline \multicolumn{7}{c}{$\mathbf{c}$} \\
\hline No. & $\delta_{\mathrm{H}}($ mult., $J$ in Hz) & $\delta_{\mathrm{C}}$ & No. & $\delta_{\mathrm{H}}($ mult., $J$ in Hz & $\delta_{\mathrm{C}}$ \\
\hline 1 & & $114.7, \mathrm{C}$ & $1^{\prime}$ & & $126.7, \mathrm{C}$ \\
2 & $159.5, \mathrm{C}$ & $2^{\prime}$ & & $157.7, \mathrm{C}$ \\
3 & $6.65, \mathrm{~d}(8.4)$ & $104.9, \mathrm{CH}$ & $3^{\prime}$ & $6.98, \mathrm{~d}(7.8)$ & $111.9, \mathrm{CH}$ \\
4 & $7.18, \mathrm{~d}(8.4)$ & $129.1, \mathrm{CH}$ & $4^{\prime}$ & $7.24, \mathrm{td}(7.8,1.5)$ & $129.5, \mathrm{CH}$ \\
5 & $6.65, \mathrm{~d}(8.4)$ & $104.9, \mathrm{CH}$ & $5^{\prime}$ & $6.99, \mathrm{t}(7.8,7.6)$ & $127.3, \mathrm{CH}$ \\
6 & & $159.5, \mathrm{C}$ & $6^{\prime}$ & $7.51, \mathrm{dd}(7.6,1.5)$ & $127.5, \mathrm{CH}$ \\
7 & $6.96, \mathrm{~d}(16.2)$ & $123.4, \mathrm{CH}$ & $7^{\prime}$ & $6.99, \mathrm{~d}(16.1)$ & $123.4, \mathrm{CH}$ \\
8 & $6.76, \mathrm{dt}(16.2,6.0)$ & $131.0, \mathrm{CH}$ & $8^{\prime}$ & $6.38, \mathrm{dt}(16.1,6.0)$ & $128.1, \mathrm{CH}$ \\
$9 / 9^{\prime}$ & $4.19, \mathrm{dt}(6.0,1.4)$ & $73.0, \mathrm{CH}_{2}$ & $9^{\prime}$ & $4.19, \mathrm{dt}(6.0,1.4)$ & $71.4, \mathrm{CH}_{2}$ \\
$2-\mathrm{OCH}_{3}$ & $3.84, \mathrm{~s}$ & $56.1, \mathrm{CH}_{3}$ & $2^{\prime \prime}-\mathrm{OCH}_{3}$ & $3.84, \mathrm{~s}$ & $55.8, \mathrm{CH}_{3}$ \\
$6-\mathrm{OCH}_{3}$ & $3.84, \mathrm{~s}$ & $56.1, \mathrm{CH}_{3}$ & & & \\
\hline
\end{tabular}

The HMBC correlations from the three aromatic methoxys $\left(\delta_{\mathrm{H}} 3.84\right)$ to the oxygenated aromatic carbons $\left(\delta_{\mathrm{C}} 159.5 \times 2,157.7\right)$ indicated the presences of a 1,3-dimethoxy-1,2,3-tri substituted and a 2-methoxy-1,2-disubstituted benzene rings (Figure 2). The COSY spectrum indicated the ${ }^{1} \mathrm{H}-{ }^{1} \mathrm{H}$ spin systems $\left(\mathrm{H}-7 / \mathrm{H}-8 / \mathrm{H}_{2}-9\right.$ and $\left.\mathrm{H}-7^{\prime} / \mathrm{H}^{-} 8^{\prime} / \mathrm{H}_{2}-9^{\prime}\right)$ for two propenyl moiety. Additional HMBC correlations from $\mathrm{H}-7\left(\delta_{\mathrm{H}} 6.96\right)$ and $\mathrm{H}^{-7}{ }^{\prime}\left(\delta_{\mathrm{H}} 6.99\right)$ to $\mathrm{C}-1$ and $\mathrm{C}-1^{\prime}$ assigned an 1,3-dimethoxy cinnamyl and a 2-methoxy cinnamyl moieties. And these two cinnamyl moieties were connected via a ether linkage by the HMBC correlations from H-9 to C-9' and H-9' to C-9. Thus, the structure of 1 was established as depicted and was named aspeverether A. Compound 1 possessed an unusual 1,3dimethoxy-2-vinylbenzene moiety, which is rarely found in nature.

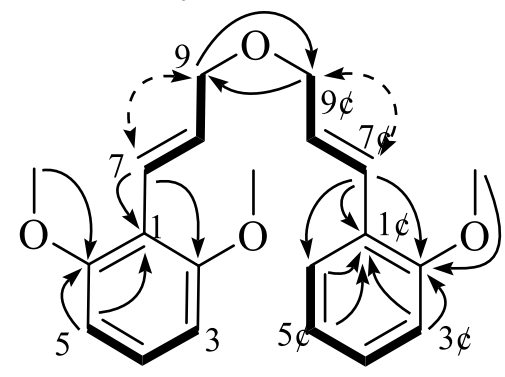

Figure 2. ${ }^{1} \mathrm{H}-{ }^{1} \mathrm{H} \operatorname{COSY}(-), \operatorname{HMBC}(\rightarrow)$, and $\operatorname{NOE}\left({ }^{\star}-{ }^{\star}\right)$ correlations of 1.

Besides, the known compounds were established as O-methoxy cinnamaldehyde (2) [12], 2,6dimethoxy cinnamaldehyde (3) [13], altechromone A (4) [14], saccharonol A (5) [15], sulochrin (6) [16], questinol (7) [17], 7-hydroxyemodin (8) [18] by comparing their ${ }^{1} \mathrm{H}$ and ${ }^{13} \mathrm{C}$ NMR data with reported data in the literature.

Compounds 1-8 were tested for their inhibitory activities against $\alpha$-glucosidase. As results, compound $\mathbf{6}$ exhibited an $\mathrm{IC}_{50}$ value of $71 \mu \mathrm{M}$, which is more active than the positive control acarbose $(210 \mu \mathrm{M})$. And other compounds showed inhibitions less than $30 \%$ at the concentration of $200 \mu \mathrm{M}$.

\section{Supporting Information}

Supporting Information accompanies this paper on http://www.acgpubs.org/journal/records-ofnatural-products

\section{ORCID}

Rong Yao: 0000-0002-0015-7396

Shen Yao: $0000-0001-6731-0255$

Ailian Hua: $\underline{0000-0003-1453-1112}$ 


\section{References}

[1] J. Wang, W. He, X. Huang, X. Tian, S. Liao, B. Yang, F. Wang, X. Zhou and Y. Liu (2016). Antifungal new oxepine-containing alkaloids and xanthones from the deep-sea-derived fungus Aspergillus versicolor SCSIO 05879, J. Agric. Food Chem. 64, 2910-2916.

[2] K. Nakanishi, M. Doi, Y. Usami, T. Amagata, K. Minoura, R. Tanaka, A. Numata and T. Yamada (2013). Anthcolorins A-F, novel cytotoxic metabolites from a sea urchin-derived Aspergillus versicolor, Tetrahedron 69, 4617-4623.

[3] Z. Cheng, D. Liu, W. Cheng, P. Proksch and W. Lin (2018). Versiquinazolines L-Q, new polycyclic alkaloids from the marine-derived fungus Aspergillus versicolor, RSC Adv., 8, 31427-31439.

[4] Y. Zhuang, X. Teng, Y. Wang, P. Liu, H. Wang, J. Li, G. Li and W. Zhu (2011). Cyclopeptides and polyketides from coral-associated fungus, Aspergillus versicolor LCJ-5-4, Tetrahedron 67, 7085-7089.

[5] J. Peng, H. Gao, X. Zhang, S. Wang, C. Wu, Q. Gu, P. Guo, T. Zhu and D. Li (2014). Psychrophilins E$\mathrm{H}$ and versicotide $\mathrm{C}$, cyclic peptides from the marine-derived fungus Aspergillus versicolor ZLN-60, J. Nat. Prod. 77, 2218-2223.

[6] R. Chen, Z. Cheng, J. Huang, D. Liu, C. Wu, P. Guo and W. Lin (2017). Versicotides D-F, new cyclopeptides with lipid-lowering activities, RSC Adv., 7, 49235-49243.

[7] Y. M. Lee, H. Li, J. Hong, H. Y. Cho, K. S. Bae, M. A. Kim, D.-K. Kim and J. H. Jung (2010). Bioactive metabolites from the sponge-derived fungus Aspergillus versicolor, Arch. Pharmacal Res. 33, 231-235.

[8] W. Lin, G. Brauers, R. Ebel, V. Wray, A. Berg, Sudarsono and P. Proksch (2003). Novel chromone derivatives from the fungus Aspergillus versicolor isolated from the marine sponge Xestospongia exigua, J. Nat. Prod. 66, 57-61.

[9] H. Gao, L. Zhou, S. Cai, G. Zhang, T. Zhu, Q. Gu and D. Li (2013). Diorcinols B-E, new prenylated diphenyl ethers from the marine-derived fungus Aspergillus versicolor ZLN-60, J. Antibiot., 66, 539542 .

[10] Z. Wu, Y. Wang, D. Liu, P. Proksch, S. Yu and W. Lin (2016). Antioxidative phenolic compounds from a marine-derived fungus Aspergillus versicolor, Tetrahedron 72, 50-57.

[11] Z. Cheng, Y. Li, W. Liu, L. Liu, J. Liu, W. Yuan, Z. Luo, W. Xu and Q. Li (2019). Butenolide derivatives with $\alpha$-glucosidase inhibitions from the deep-sea-derived fungus Aspergillus terreus YPGA10, Marin. Drugs 17, 332.

[12] D. T. L. Huong, Y. S. Jo, M. K. Lee, K. H. Bae and Y. H. Kim (2000). Monoamine oxidase inhibitors from Cinnamomi Cortex, Nat. Prod. Sci. 6, 16-19.

[13] K. V. Rao and J. H. Johnson (1998). Occurrence of 2,6-dimethoxy cinnamaldehyde in taxus floridana and structural revision of taxiflorine to taxchinin M, Phytochemistry 49, 1361-1364.

[14] L. Liu, W. Xu, S. Li, M. Chen, Y. Cheng, W. Yuan, Z. Cheng and Q. Li (2019). Penicindopene A, a new indole diterpene from the deep-sea fungus Penicillium sp. YPCMAC1, Nat. Prod. Res. 33, 2988-2994.

[15] G. Du, Z.C. Wang, Y.K. Yang, H.M. Yang, H.Y. Yang, M. Zhou, Y.Q. Ye, X.M. Li and Q.F. Hu (2015). New isocoumarins from the fermentation products of entophytic fungus Aspergillus versicolor and their anti-tobacco mosaic virus activities, Heterocycles 91, 1996-2002.

[16] D. Liu, L. Yan, L. Ma, Y. Huang, X. Pan, W. Liu and Z. Lv (2015). Diphenyl derivatives from coastal saline soil fungus Aspergillus iizukae, Arch. Pharm. Res. 38, 1038-1043.

[17] D. Zhao, F. Cao, X.-J. Guo, Y.-R. Zhang, Z. Kang and H.-J. Zhu (2018). Antibacterial indole alkaloids and anthraquinones from a sewage-derived fungus Eurotium sp., Chem. Nat. Compd ${ }^{+}$. 54, 399-401.

[18] G. Bringmann, G. Lang, S. Steffens, E. Günther and K. Schaumann (2003). Evariquinone, isoemericellin, and stromemycin from a sponge derived strain of the fungus Emericella variecolor, Phytochemistry 63, 437-443.

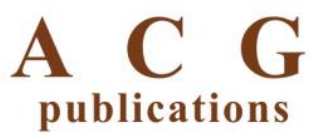

(C) 2020 ACG Publications 